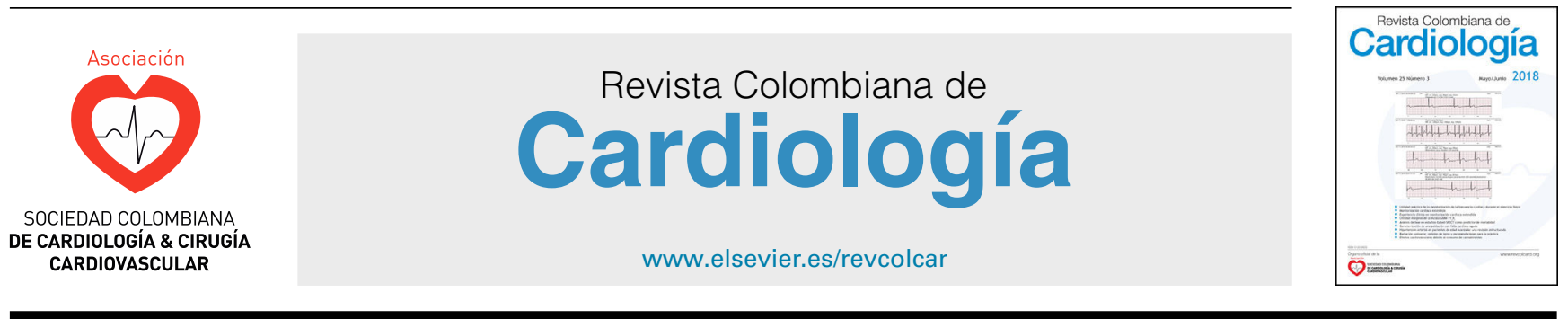

\title{
Ética en las relaciones médicas con la industria farmacéutica
}

\author{
Diana P. Martínez
}

Grupo de Trabajo de Bioética Clínica, Sociedad Colombiana de Cardiología y Cirugía Cardiovascular. Bogotá Colombia

Recibido el 10 de abril de 2019; aceptado el 11 de abril de 2019

Utopía: "el sueño de unos pocos que se convierte en la pesadilla de los demás",

Fernando Savater

Es innegable que la relación de los profesionales médicos con la industria farmacéutica, al igual que toda actividad humana, está sometida a una serie de tensiones, que, de acuerdo con su evolución, pueden conducir a un número considerable de dilemas éticos en la práctica médica ${ }^{1}$.

Se entiende por dilema ético aquella situación que compete a la naturaleza moral, es decir, aquella que plantea cómo obrar bien, evitando hacer daño, valorando las consecuencias del acto y logrando obtener el mayor beneficio para la mayoría de personas involucradas, con base en unas reglas "para el bien" establecidas ${ }^{2,3}$.

Hablar de los aspectos éticos existentes en la relación que los profesionales médicos tienen con la industria farmacéutica, es recorrer un largo camino en el cual se mezclarán aspectos relacionados con la formación profesional, la naturaleza humana, los sistemas de salud de los diferentes países en los que se desarrolla la práctica médica y el desarrollo de la economía global, así como también la evolución de las diferentes unidades y proyectos de negocio de las multinacionales farmacéuticas, entre otros ${ }^{2}$.

Correo electrónico: dimartru@gmail.com
Si bien hoy es preciso aceptar que en décadas anteriores la formación continuada de los profesionales médicos había estado sujeta en gran medida a los patrocinios de la industria farmacéutica y que parte de sus presupuestos era destinado a la formación médica, pero también a la "atención", en ocasiones desmedida, de los mismos, cada vez más estos patrocinios están sujetos al desarrollo actual de políticas estrictas del gasto en las diferentes compañías de la industria farmacéutica. Para algunos más que para otros, es familiar que en años anteriores los gastos de la industria farmacéutica trascendían a ofrecimientos $y$ atenciones exageradas y por fuera de lo estrictamente relacionado con la educación continuada, dando como resultado el origen a contratos tácitos de prescripción favorecida, entre otros. Es por eso que hoy una relación equilibrada y transparente entre los profesionales médicos y la industria farmacéutica, es, sin lugar a dudas, el mayor reto al que se ven enfrentados las sociedades médicas científicas, los sistemas políticos de salud y las políticas del gasto de la industria farmacéutica. De ahí que la formación ética se convierta en el motor que impulsa el desarrollo de actitudes y lineamientos responsables, tanto de los profesionales médicos como de la industria farmacéutica, transformándolos en una invitación al respeto por la autonomía de las decisiones y elecciones médicas, basadas en conocimientos académicos y acordes con las políticas y lineamientos de salud en el escenario donde se desarrolle su actividad profesional ${ }^{3-5}$. 
Es aquí donde me permito citar a Rogelio Altisent (Director de la cátedra de Profesionalismo y Ética Clínica de la Universidad de Zaragoza, presidente de la Comisión Central de Deontología de la Organización Médica Colegial de España de 2006 a 2009 y presidente del Comité de Bioética de Aragón desde 2013), "El Desarrollo Profesional Continuado es un proceso que debe acompañar las sucesivas etapas de la vida del profesional, con sus distintos momentos de motivación y crisis, y conviene entender su fundamentación ética, reflexionando sobre la calidad asistencial. Los profesionales que cuidan pacientes también necesitan recibir atención para diagnosticar y superar las situaciones de inercia, ausencia de iniciativa, escepticismo y falta de autocrítica. Sólo se puede garantizar la calidad asistencial si los profesionales desarrollan un satisfactorio progreso de su carrera, para lo cual es necesario que los servicios de personal y de recursos humanos de las instituciones sanitarias dediquen sus mejores energías a cuidar la promoción de sus profesionales" 6 .

En otras palabras, es la formación médica continuada, la que puede darle a los profesionales médicos las herramientas necesarias para iniciar y mantener una relación correcta con la industria farmacéutica, fortaleciendo las limitaciones propias e innegables de la condición de seres humanos, entre las cuales se encuentran la racionalidad, el egoísmo y la persecución innata de oportunidades, todo aunado a las hoy innumerables limitaciones de los sistemas políticos de atención en salud, los cuales promueven la desmotivación profesional y dejan por fuera de sus presupuestos la formación médica continuada, los programas de investigación y el desarrollo de nuevas políticas de atención sanitaria, estableciendo una gran brecha entre la actividad médica asistencial y las políticas de salud ${ }^{4,7-9}$.

De manera histórica, la brecha a la que se hace referencia, ha llevado a que haya grandes vacíos en la motivación y formación de los profesionales médicos y a que estos, a lo largo de muchos años, hayan podido ser "utilizados" por la industria farmacéutica para interceptar de manera discreta y en ocasiones no tanto, su autonomía, induciendo y desviando la prescripción de fármacos y dispositivos médicos entre otros, debido al conflicto de intereses desarrollado por las "deudas" adquiridas en atenciones, regalos y favores recibidos, a través de patrocinios de formación médica ${ }^{1,5,9}$.

De igual manera, los diferentes protocolos de investigación pueden exponer a situaciones no menos preocupantes a las sociedades médicas científicas, en lo que se refiere a la publicación de resultados beneficiosos para la industria patrocinadora y la omisión o subvaloración de eventos adversos unidos a la no atención del respeto por la autonomía de los pacientes, con una sobreestimación frecuente del consentimiento informado y la ausencia del respeto por la justicia distributiva de los recursos en salud. Los resultados favorecedores pueden determinar la prescripción de productos innovadores de la industria farmacéutica y de esta manera aumentar la "percepción" de necesidades terapéuticas y biotecnológicas en salud ${ }^{9,10}$.

Hoy se sabe claramente que el desarrollo de nuevos productos farmacéuticos y de tecnología médica, no tiene un crecimiento lineal con relación a los presupuestos y políticas de salud, que permiten la aplicación y el pago de los mismos. Por esta razón, la práctica médica cotidiana entra día a día en un mayor conflicto ante la escasez de recursos económicos, incluso en los países con economías estables, que permitan hacer propias todas las tecnologías innovadoras que presenta la industria farmacéutica, que, conviene recordar, son una de las industrias económicas más grandes del mundo 8,11 .

A estas diferentes situaciones se ven enfrentados continuamente los profesionales médicos y en medio de sus dificultades y limitaciones, aparecen las políticas comerciales de la poderosa industria farmacéutica, para dar un tentador y peligroso alivio a sus necesidades. En esta, la industria farmacéutica también se plantea un gran dilema entre su razón social de existir, es decir, contribuir al descubrimiento e innovación que permita mejorar la prevención, tratamiento y rehabilitación de las enfermedades y de esta manera la calidad de vida de las diferentes poblaciones y la obligación de generar utilidades por su naturaleza comercial, lo cual se convierte en un enemigo de su objetividad ${ }^{9,10}$.

Es por esto que la invitación a una relación equilibrada y transparente entre los profesionales médicos y la industria farmacéutica, hace evidentes todas estas limitaciones, para que dentro del conocimiento de las mismas, con argumentación y racionamiento críticos de ambas partes, éstas puedan ser reconocidas y enfrentadas de manera profesional y ética, y se logre mantener el respeto por la dignidad del quehacer de la profesión médica, junto al cumplimiento de las políticas globales de salud y al crecimiento económico de la industria farmacéutica?'

El fundamento ético de la práctica médica se expresa bajo los principios de autonomía, es decir, el derecho que tiene cada individuo de hacer parte activa de las decisiones tomadas en su proceso de salud y enfermedad, y el respeto que debe tenerse de esta participación; el principio de beneficencia, que busca de manera oportuna el bienestar del paciente, analizando la relación riesgo/beneficio de las diferentes intervenciones médicas; el principio de no maleficencia, que recuerda a los profesionales médicos no hacer daño, y, finalmente, el principio de justicia en la distribución de los recursos, que hace referencia al valor de la equidad y al uso racional de los recursos de los sistemas de salud ${ }^{12}$.

Así mismo, la práctica médica debe alejarse de los escenarios que muestren incentivos desproporcionados, solicitudes y peticiones inapropiadas que ponen en riesgo la autonomía del servicio médico y el bienestar de los pacientes. De igual manera, aunque en ocasiones puede resultar complicado, la comunidad médica debe estar atenta a las "estrategias comerciales" disfrazadas, en las se induce la prescripción de determinados productos, interceptando de forma directa el criterio médico y la integridad profesional $^{1,9}$.

Cada día ofrece un nuevo reto dentro de la relación de los profesionales médicos y la industria farmacéutica, porque cada día habrá una y muchas decisiones por tomar y detrás de cada una de estas, un riesgo para el paciente, para la autonomía de la práctica médica, para el manejo responsable de los beneficios económicos derivados del oficio médico y para los objetivos económicos de la industria y de los sistemas políticos de salud. Esto obliga a que cada día, los profesionales médicos y la industria farmacéutica, pongan su atención en estos temas y en especial, los que se refieren a la educación médica continuada y al desarrollo de investigaciones $^{12}$. 
Debido a que en la práctica médica actual, las políticas de eficiencia y productividad de los servicios de salud no contemplan dar oportunidad a los procesos de educación continuada y que estos cada vez son más costosos, las universidades y las instituciones prestadoras de los servicios de salud tienen la gran responsabilidad de proporcionar ambientes neutros de formación crítica académica y de conformar grupos de investigación que sean dueños de la iniciativa de generar nuevos conocimientos, que de manera oportuna den respuesta a las diferentes necesidades locales de la comunidad médica y de la sociedad a la que dirigen su trabajo. Además, deberían lograr espacios políticos, que les permitan intervenir de manera activa en las diferentes políticas de salud que han dejado de lado la formación continuada de los profesionales de salud, desarrollando gran desmotivación e inercia en el trabajo médico. Así mismo, las universidades e instituciones prestadoras de los servicios de salud, al recibir patrocinio por parte de la industria farmacéutica, para la realización de eventos académicos, deberán tener límites claros, previamente establecidos en cuanto a la participación de ésta, en temas académicos. De esta manera la industria farmacéutica debería cada vez más implementar políticas claras y trasparentes para la utilización adecuada de sus recursos en el tema de investigación y patrocinios de educación médica continuada ${ }^{6,9,11-13}$.

De otro lado, si cada profesional médico, desde su individualidad, quiere conservar su autonomía e independencia profesional, deberá mantenerse alejado de convenios con la industria farmacéutica que le generen preocupación, si fuesen conocidos por la comunidad médica en general. Éste debe mantenerse alejado del pago directo, en dinero o especie, por parte de la industria farmacéutica, relacionado con una prescripción determinada de un producto o dispositivo o con eventos "pseudo" científicos ${ }^{6,9}$.

Por último, aunque la relación de la industria farmacéutica y los profesionales médicos ha sido una histórica y por muchos años en nombre de las estrategias comerciales, se han realizado prácticas inmorales frente al ejercicio médico y la prescripción favorecida de algunos productos, no por ser frecuente incluso en este tiempo, estas prácticas deben ser aceptadas. La evolución de la formación médica integral debe llevar a toda la comunidad profesional a defender y mantener en alto su autonomía y dignidad profesional.

\section{Bibliografía}

1. Brody H. Clarifying conflict of interest. Am J Bioethics. 2011;11:23-8.

2. Keller F, Marczewski K, Pavlović D. The relationship between the physician and pharmaceutical industry: background ethics and regulation proposals. Croat Med J. 2016;57:398-401, http://dx.doi.org/10.3325/cmj.2016.57.398.

3. DeJong C, Dudley A. Reconsidering physician pharmaceutical industry relationships. JAMA. 2017;317, 1772/3.

4. Suresh M, Kamala R. Championing ethics. Industry Viewpoint. 2013;4:33-8.

5. Gray GC. The Ethics of Pharmaceutical Research Funding: A Social Organization Approach. J Law Med Ethics. 2013;41:629-34. Fall.

6. Altisent R. La ética del desarrollo profesional continuado en la promoción de la calidad asistencial. Acta Bioethica. 2010;16:155-64.

7. Martínez Bullé Goyri V. Bioética, derecho y derechos humanos En: Perspectivas de Bioética. México. Universidad Nacional Autónoma de México. Facultad de Filosofía y Letras. Comisión Nacional de los Derechos Humanos. FCE. 2008.

8. De la Garza MT. Bioética y Biopolítica Perspectivas de Bioética. México. Universidad Nacional Autónoma de México. Facultad de Filosofía y Letras. Comisión Nacional de los Derechos Humanos. FCE. 2008.

9. Gómez A, Latorre C, Carreño JN. Dilemas éticos en las relaciones entre la industria farmacéutica y los profesionales de la salud. Persona y Bioética. 2007;11:23-38.

10. Orlowski JP, Wateska L. The effects of pharmaceutical Firm enticements on physician prescribing patterns. There's no such thing as a free lunch. Chest. 1992;102:270-3.

11. Shaw B, Whitney P. Ethics and compliance in global pharmaceutical industry marketing and promotion: The role of the IFPMA and self-regulation. Pharmaceuticals Policy and Law. 2016;18:199-206, 10.3233/PPL-160443.

12. Niteesh KCh, Stelfox HTh, Detsky AS. Relationships between authors of clinical practice guidelines and the pharmaceutical industry. JAMA. 2002;287:612-7.

13. Gracia D. Profesión médica, investigación y justicia sanitaria. Colección Ética y Vida, 4. Bogotá: Editorial El Búho Ltda; 1998. 